\title{
Magic numbers of nanoholes in graphene: Tunable magnetism and semiconductivity
}

\author{
X. Y. Cui, ${ }^{1}$ R. K. Zheng, ${ }^{1}$ Z. W. Liu, ${ }^{1}$ L. Li, ${ }^{1}$ B. Delley, ${ }^{2}$ C. Stampfl, ${ }^{3}$ and S. P. Ringer ${ }^{1}$ \\ ${ }^{1}$ Australian Centre for Microscopy \& Microanalysis, The University of Sydney, New South Wales 2006, Australia \\ ${ }^{2}$ Paul Scherrer Institut, WHGA/123, CH-5232 Villigen PSI, Switzerland \\ ${ }^{3}$ School of Physics, The University of Sydney, New South Wales 2006, Australia
}

(Received 4 July 2011; published 6 September 2011)

\begin{abstract}
Patterned vacancy clusters (or nanoholes) can modify the electronic structure of graphene, and thereby generate entirely new functionalities. Knowledge of the relative stability of various nanoholes and associated properties is essential for the rational design and fabrication of practical devices. Extensive first-principles results reveal remarkable stability in certain ring configurations, as well as modified triangular and hexagonal vacancy configurations. The identified magic numbers of vacancies are 2, 4, 6, 28, 39, 42, 52, and 60. A large number of the nanoholes exhibit magnetic states with diverse energy band-gap values. Some large nanoholes possess nonzero net moments in all possible magnetic solutions, showing that the corresponding magnetization is robust against thermal fluctuation. Room-temperature ferromagnetism in graphene (and graphite) can be attributed to the local ferri- or ferromagnetism in large nanoholes, which can be created under irradiation and chemical treatment. Nanohole-induced, stable magnetic-semiconducting graphene is expected to be useful in graphene-based spintronics.
\end{abstract}

DOI: 10.1103/PhysRevB.84.125410

PACS number(s): 73.22.Pr, 71.15.Mb, 71.15.Nc, 75.50.Dd

\section{INTRODUCTION}

Graphene-based materials have attracted great attention since the successful isolation of graphene in 2004. ${ }^{1}$ Pure graphene, an atomically thin layer of $s p^{2}$ hybridized carbon, exhibits truly spectacular structural, mechanical, electronic, thermal, and possibly magnetic properties, and these hold promise for a vast range of nanotechnologies. ${ }^{2-4}$ However, the presence and evolution of atomic-scale defects, ${ }^{5,6}$ such as vacancies, has profound and often undesirable consequences on the mechanical ${ }^{7}$ and transport properties. ${ }^{8-11}$ On the other hand, as in conventional semiconductors, ${ }^{12}$ the selective introduction of particular defects can be used to modify the electronic properties in graphene and related materials. One of the most important types of defects is atomic vacancies, which have been proposed as host sites of functional groups and catalytic sites. ${ }^{13}$ Regularly spaced holes (antidots) are also proposed for electron-spin qubits. ${ }^{14}$

Currently there is a surge in the attempts to use patterned vacancies (nanomeshes) into extended structures to achieve entirely new properties in graphene-based materials, particularly for band-gap engineering - changing the semimetal graphene to be semiconducting ${ }^{15-20}$ or a metallic wire..$^{21}$ Emerging information indicates that the resulting properties are sensitive to the local size, shape, and structure or architecture of the nanoholes. Precise atomic-fabrication techniques will be needed to achieve targeted properties; however, even then, the edge atoms of nanoholes may undergo rearrangement and reconstruction, changing the local architecture and therefore the associated electronic properties. ${ }^{6,22}$ Hence, a systematic study of the stability of various nanoholes and their associated properties represents a crucial step toward understanding and expanding the graphene materials for potential applications.

Vacancies are also intimately associated with carbon magnetism, ${ }^{23-25}$ which is an unexpected combination of small magnetization and high Curie temperatures $\left(T_{\mathrm{C}}\right)$ that are considered to be intrinsic ${ }^{26}$ and due to vacancies, such as those that are induced by electron or ion irradiation in graphite. ${ }^{24,26-32}$ Recently, room-temperature ferromagnetism (RTFM) was observed in graphene prepared by the chemical preparation method, ${ }^{33}$ which is believed to include a significant amount of topological defects, including nanosized hole regions. ${ }^{34}$ The possibility of magnetism in pure carbon systems is of fundamental and technological importance. Because of the small spin-orbit coupling, long spin scattering length, and ballistic transport characteristics, the possibility of magnetic graphene provides a great arena to develop the spin-polarized devices. ${ }^{35}$

Theoretically, while local magnetism in graphene sheets has been well established, ${ }^{23,36,37}$ there remains substantial uncertainty concerning the defect structures and how these defects interact to result in a strong ferromagnetism with high $T_{\mathrm{C}}$. Most theoretical attempts to explain the origin of the RTFM have focused on the interaction between single or some specific vacancy clusters, ${ }^{38-41}$ usually containing hydrogen atoms in the graphene lattice. ${ }^{23,36,39-46}$ These models explicitly require a high vacancy density so as to exceed the percolation threshold for a collective ferromagnetism. ${ }^{47} \mathrm{We}$ argue, however, that these scenarios may be unlikely due to the high formation energy of single carbon vacancy and low stability of some proposed specific vacancy clusters - an issue highlighted in defect-induced magnetism in oxides ${ }^{48}$ Indeed, Vozmediano et al. ${ }^{49}$ concluded that $T_{\mathrm{C}}$, assuming a random distribution of single local moments, is as low as $1 \mathrm{~K}$. Under irradiation, the density of the defect will increase. However, we argue, and demonstrate below, that this will more likely create large vacancy clusters, or nanosized void regions (nanoholes), as was observed in experiments. ${ }^{22,34,50}$ Another mechanism of magnetic carbon concerns edge-induced (particularly the zigzag edge) magnetism in graphene nanostructures ${ }^{51-53}$ (such as ribbons, flakes, and islands). However, the magnetic interaction is typically rather weak. Recently, it has been argued that magnetic edge states in zigzag-edge graphene nanoribbons might not exist or might not be stable at room 
temperature in real systems. ${ }^{54}$ So the question remains open: Is it possible to realize RTFM in pure carbon systems?

The motivation behind this paper is twofold: First, through comprehensive total energy calculations, this work aims to unravel the structures and energetics of various differentsized graphene nanoholes. In particular, by comparing the formation energies and dissociate energies, we aim to identify the stable nanohole architectures-certain magic numbers of vacancies-that can impart novel and technologically significant properties. Second, this work reports a scenario of native magnetism in graphene (and graphite). Our results show that the size of the multivacancy tends to increase to form large nanoholes, as observed in experiments under irradiation or chemical treatment. ${ }^{22,34} \mathrm{We}$ attribute the experimentally reported RTFM to the ferri- or ferromagnetic large nanoholes, which also exhibit diverse band-gap values in graphene. The combination of tunable semiconductivity and strong ferromagnetism in patterned or nanostructured graphene would present new opportunities in spintronics.

\section{MODEL}

We performed all-electron, spin-polarized density functional theory (DFT) calculations using the generalized gradient approximation ${ }^{55}$ with the periodic $\mathrm{DMOL}^{3}$ code. $^{56}$ The wave functions were expanded in terms of a double-numerical quality, localized basis set with a real-space cutoff of $9 \mathrm{bohr}$. Polarization functions and scalar-relativistic corrections were incorporated. Single-layer graphene was simulated using a large supercell of 288 atoms $(a=29.64, b=25.64$, and $c=22.55 \AA$ ). Reciprocal-space k-point meshes of $1 \times 1 \times 2$ were used. We allowed full atomic relaxation where the forces on the atoms were less than $0.005 \mathrm{eV} / \AA$.

Although hydrogenation is proposed to enhance the magnetism, ${ }^{36,44,46}$ to address the possibility of pure-carbon magnetism, we did not saturate the dangling bonds with hydrogen atoms. Such a choice was further inspired by the experimental finding that the edge atoms of nanoholes were not terminated by hydrogen or other functional groups. ${ }^{22}$ For a given-sized nanohole, several geometrical configurations were considered to determine the most stable structure. Moreover, for each structure, the subtle competition between paramagnetic, ferromagnetic, and various ferrimagnetic or antiferromagnetic states was carefully examined.

We used unified quantities, i.e., the formation energy and dissociation energy, to evaluate the relative stability of different nanoholes. The formation energy $E_{f}$ is calculated as

$$
E_{f}=E_{d}-E_{p}+n \times \mu_{\mathrm{C}},
$$

where $E_{d}$ and $E_{p}$ are the total energies of the defective and perfect graphene supercell, respectively. $\mu_{\mathrm{C}}$ is the chemical potential of the carbon atom, which is determined using relaxed graphite. $n$ is the number of carbon vacancy, i.e., the size of the nanohole. The dissociation energy of the $n$-site vacancy is defined in two ways,${ }^{57}$ i.e.,

$$
\begin{aligned}
& D_{1}(n)=E_{f}(n-1)+E_{f}(1)-E_{f}(n), \\
& D_{2}(n)=E_{f}(n+1)+E_{f}(n-1)-2 E_{f}(n),
\end{aligned}
$$

where $E_{f}(n)$ is the formation energy of the $n$-site vacancy.
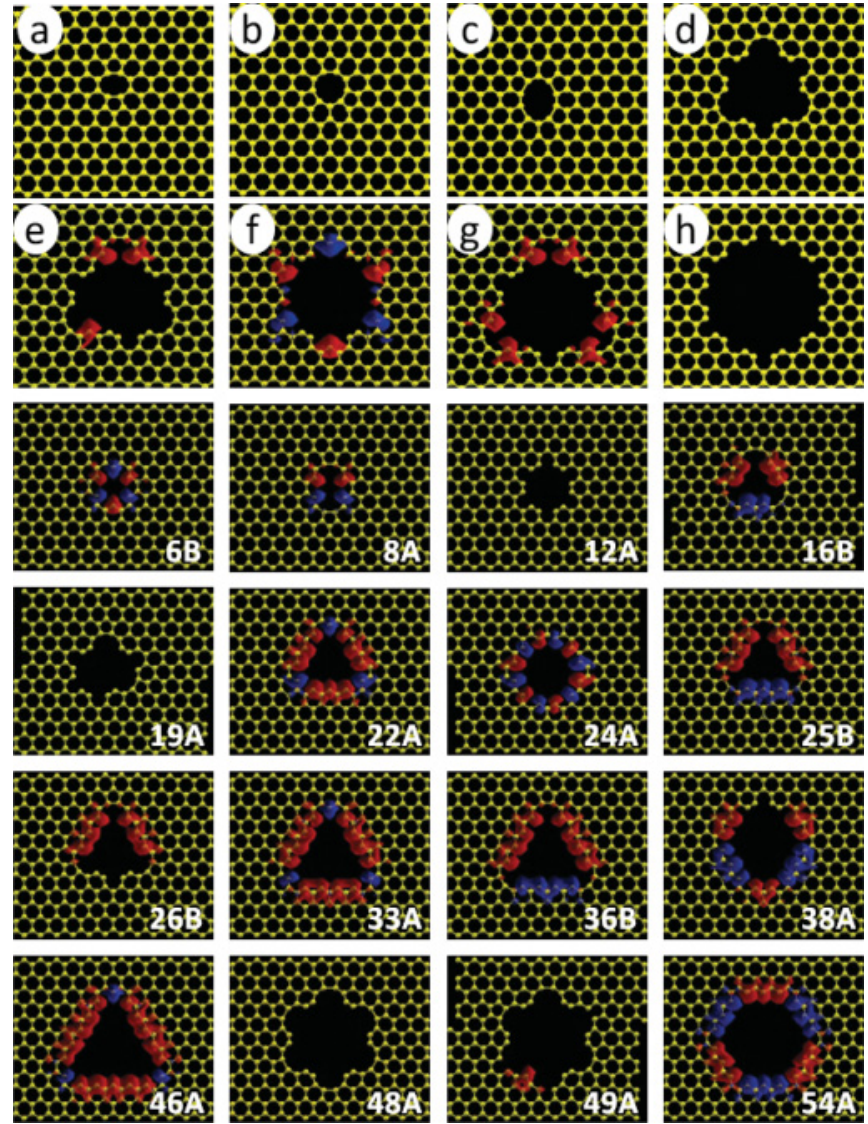

FIG. 1. (Color online) Atomic and magnetic structures for some selected (stable and all those that are discussed in the text) nanoholes. The predicted magic configurations with high degrees of stability are (a) 2A, (b) 4A, (c) 6A, (d) 28A, (e) 39A, (f) 42A, (g) 52A, and (h) 60A Structures 2A, 4A, 6A, 12A, 19A, 48A, and 60A are nonmagnetic. Red (blue) isosurfaces denote positive (negative) spin polarization. The isosurface value is 0.015 electrons $/ \AA^{3}$.

\section{RESULTS AND DISCUSSION}

We present some selected nanohole structures in Fig. 1, while all the unrelaxed and relaxed structures considered in this study are shown in Supplemental Fig. $1 S .^{58}$ Energy minimization causes significant atomic reconstruction to reduce the number of dangling bonds, and hence the total energies. A general feature is that a hexagon missing one atom tends to undergo edge closure, which is in good agreement with electron microscopy 6,22 and theoretical calculations for small vacancy clusters. ${ }^{19,57,59}$ To obtain the dissociation energies, we emphasize that it is essential to consider the growth pathways of the nanoholes, which are constructed by a bottom-up approach, as detailed in Supplemental Fig. $2 \mathrm{~S} .{ }^{60}$ The governing rules for these pathways are following structural similarity and lowest energy. The calculated formation energy per vacancy and the dissociation energies $\left(D_{1}\right.$ and $\left.D_{2}\right)$ are charted as a function of nanohole size in Figs. 2 and 3, respectively.

From Fig. 2, the formation energy per vacancy decreases as the nanohole size increases, changing from $\sim 7.5 \mathrm{eV}$ for $1 \mathrm{~A}$ to $\sim 0.84 \mathrm{eV}$ for $60 \mathrm{~A}$. Interestingly, several local minima exist in the vicinity of, e.g., $n=4$ and 28 . For a given 


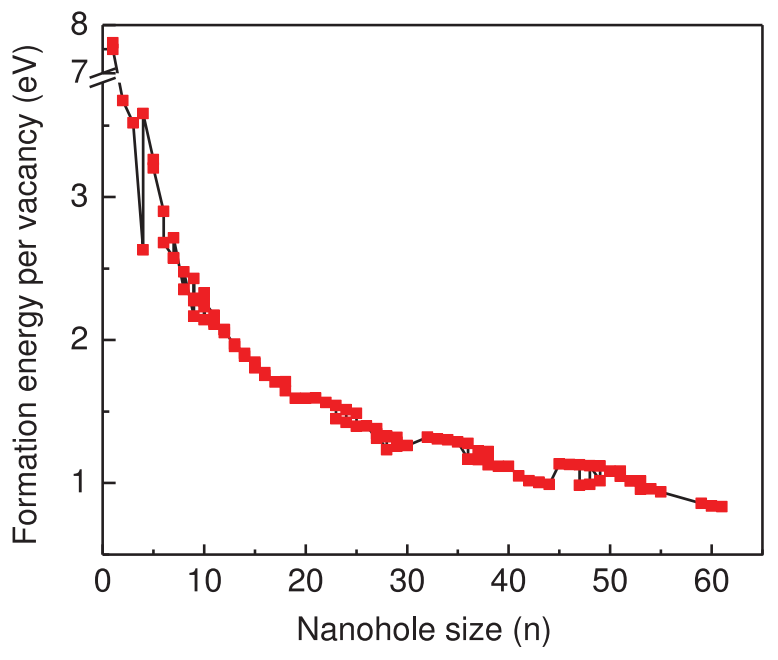

FIG. 2. (Color online) Formation energy per vacancy as a function of the nanohole size. The size is measured as the number $n$ of vacancies that constitute the nanoholes. The line is used to guide the eyes.

$n$-sized nanohole, we confirm that compact hole structures are likely to be energetically favorable. Clustering of the vacancies minimizes the number of dangling bonds and hence reduces total energy. A high-energy barrier $(\sim 7.49 \mathrm{eV})^{61}$ will greatly limit the mobility of vacancies in a graphene sheet, which means it is unlikely that nanoholes are formed through the coalescence of monovacancies. On the other hand, the continuous decrease in formation energy with nanohole size indicates that it gets progressively easier to remove edge carbon atoms and so grow the nanoholes under irradiation. Our results suggest that irradiation will more likely create large nanoholes rather than increase the density of single or small defects. We believe this explains the formation of the large nanoholes observed experimentally following irradiation ${ }^{22}$ or in chemically derived graphene. ${ }^{34}$

Consider that for a given hole structure, $D_{1}(n)$ corresponds to the dissociation into the $(n-1)$-site vacancy and monovacancy, while $D_{2}(n)$ corresponds to the dissociation into the $(n+1)$-site and $(n-1)$-site vacancies. As such, in Fig. 3,
$D_{1}(n)$ values are always positive, demonstrating the stability of the nanoholes against vacancy separation. By definition, $D_{2}(n)=D_{1}(n)-D_{1}(n+1)$, and thus a large, positive $D_{2}(n)$ value indicates the stability of a vacancy cluster against further growth. Together, $D_{1}(n)$ and $D_{2}(n)$ reveal the relative stability of a nanohole against changes in size. Here, we impose three selection criteria for a magic number: (i) a large $D_{1}(n)$ value of over $7 \mathrm{eV}$, (ii) a large $D_{2}(n)$ value of approximately $1.0 \mathrm{eV}$, and (iii) the most energetically favorable configuration for a given $n$ to stabilize the shape of a nanohole. From Fig. 2, it is evident that there exist some special structures possessing a high degree of stability: the magic numbers $n=2,4,6,28$, $39,42,52$, and 60 . The predicted stable nanoholes are shown in Fig. 1. Due to the arbitrariness of the criteria involved, it should be noted that some other configurations, such as 33A, 46A, and 48A, also possess relatively high stability.

Evidently, $D_{1}$ and $D_{2}$ show rather different trend behavior, which differs from that in Ref. 57, even though our energetically favorable structures for $n=1-8$ are in agreement with those therein. It should be noted that in Ref. 57, only one structural configuration was considered for a given-sized vacancy cluster, up to $n=8$. Moreover, the spin-polarization effect was not considered. The three small vacancy clusters $2 \mathrm{~A}$, 4A, and 6A (see Fig. 3) are the only geometries whereby each atom maintains a planar $s p^{2}$ configuration. The frequently studied triangular structures with $n=m^{2}$, where $m$ is an integer, were found to be less stable, particularly for the large nanoholes 25B, 36B, and 49A (see Fig. 1), with $D_{1}(n) \sim$ $6.6 \mathrm{eV}$, and $D_{2}(n) \sim-1.7 \mathrm{eV}$. Importantly, introducing an extra vacancy in the middle, which is the energetically preferred site, of each zigzag edge greatly enhances the stability, giving $D_{1}(n) \sim 8.2 \mathrm{eV}$ and respective $D_{2}(n)$ values of 2.86, 1.87, and $1.76 \mathrm{eV}$ for 28A, 39A, and 52A (see Fig. 3). Based on this, the modified larger triangular structures with $n=m^{2}+3$ are predicted to be stable. This finding seems in line with experimental observations showing that the zigzag edges of graphene nanoholes tend to accommodate extra vacancies. ${ }^{22}$ An alternative, though less efficient, way to enhance the stability of triangular patterns is to add one carbon atom (or eliminate one vacancy) at each corner. The resultant 22A, 33A, and $46 \mathrm{~A}$ structures (see Fig. 1) have almost unchanged $D_{1}(n)$
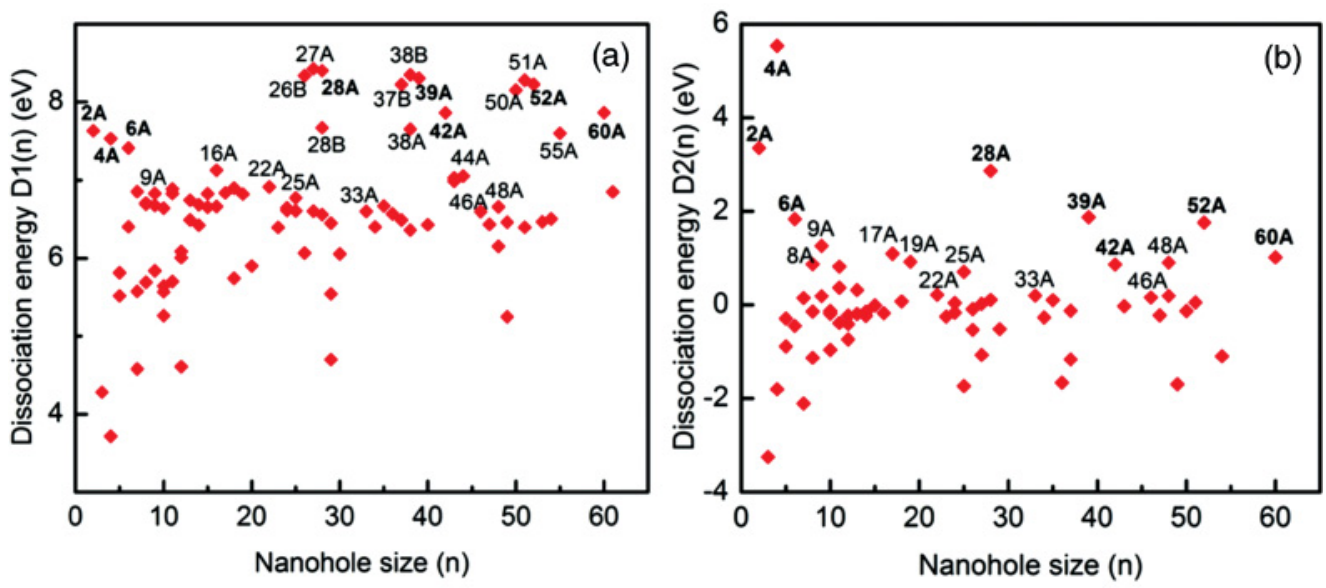

FIG. 3. (Color online) Dissociation energies (a) $D_{1}(n)$ and (b) $D_{2}(n)$ as a function of the nanohole size. Only the configurations with large dissociation values or those discussed in the text are labeled. The magic nanoholes are highlighted in bold. 

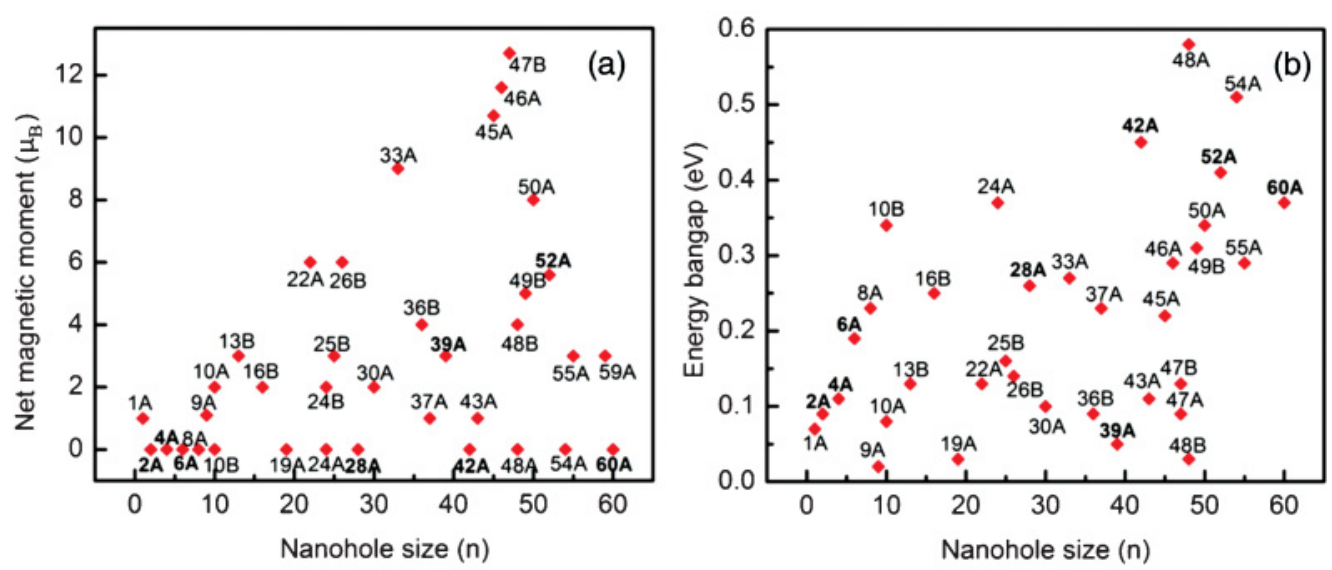

FIG. 4. (Color online) Diversity of net magnetic moment and band gap: (a) net moment and (b) calculated band-gap values as a function of nanohole size for selected configurations. The magic nanoholes are highlighted in bold.

values $(\sim 6.6 \mathrm{eV})$, but larger $D_{2}(n)$ values $(\sim 0.2 \mathrm{eV})$. Similarly, the perfect hexagons with $n=6 \times m^{2}$, corresponding to $6 \mathrm{~B}$, 24A, and 54A (see Fig. 1), possess low stabilities with $D_{1}(n)$ $\sim 6.5 \mathrm{eV}$ and respective $D_{2}(n)$ values of $-0.45,0.038$, and $-1.09 \mathrm{eV}$. By introducing three vacancies at each zigzag side of $24 \mathrm{~A}$ (or one at each side of $54 \mathrm{~A}$ ), the resultant $42 \mathrm{~A}$ ring structure (or 60A) has significantly enhanced stability, with $D_{1}(n) \sim 7.9 \mathrm{eV}$ and $D_{2}(n) \sim 1.0 \mathrm{eV}$. Our results show that changing hexagons to ringlike patterns is an effective route to enhance the stability of larger nanoholes, such as $n=96$ and 150. Overall, our results show that long zigzag edges tend to lower the stability of nanoholes. Stable structures are those with hexagonal outer edges, except 39A.
A critical finding is that magnetism and semiconductivity coexist for a large number of configurations. Figure 4 demonstrates the diversity of the calculated net magnetic moments and band-gap values. Not surprisingly, configurations 2A, $4 \mathrm{~A}$, and $6 \mathrm{~A}$, with fully $s p^{2}$-bonded atoms, are nonmagnetic. Consistent with the observation that armchair-edged graphene ribbons are nonmagnetic, ${ }^{19}$ so too are the armchair-edged ring structures 12A, 19A, 28A, 48A, and 60A (see Fig. 1). All the other configurations studied are magnetic. As expected, local atomic moments (about $0.9-1.15 \mu_{\mathrm{B}}$ ) reside mainly in the vicinity of the edge sites. Mulliken population analysis (not shown) reveals that the spin originates primarily from $C-2 p$ electrons. Introducing an extra vacancy at the zigzag
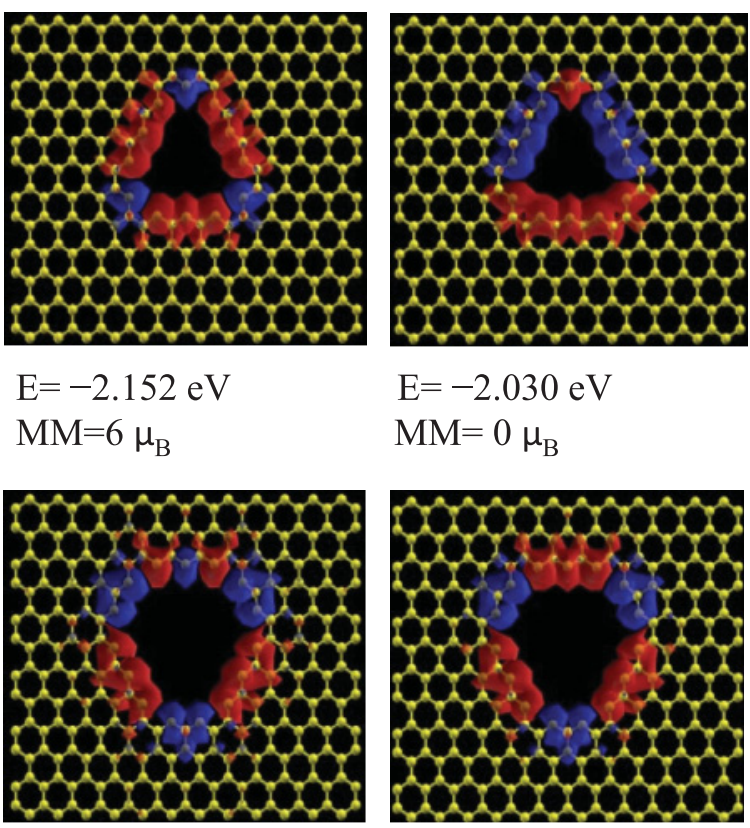

$$
\mathrm{E}=-2.721 \mathrm{eV}
$$$$
\mathrm{MM}=1 \mu_{\mathrm{B}}
$$

\begin{abstract}
$\mathrm{E}=-2.030 \mathrm{eV}$
$\mathrm{MM}=0 \mu_{\mathrm{B}}$
\end{abstract}

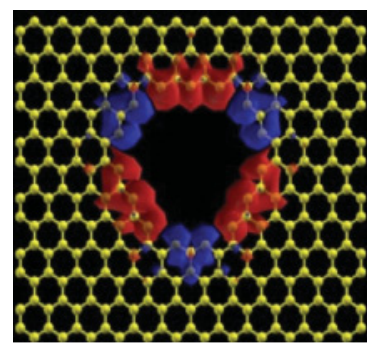

$$
\mathrm{E}=-2.715 \mathrm{eV}
$$

$\mathrm{MM}=3 \mu_{\mathrm{B}}$
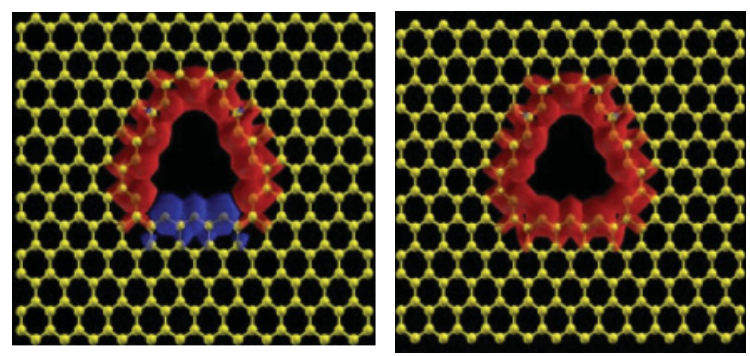

$\mathrm{E}=-2.020 \mathrm{eV}$
$\mathrm{MM}=9 \mu_{\mathrm{B}}$

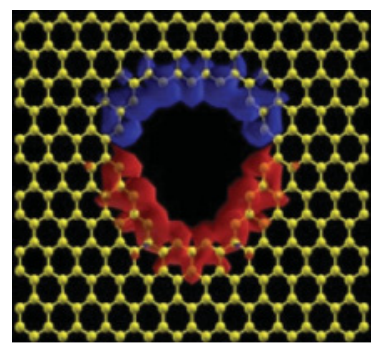

$$
\begin{aligned}
& \mathrm{E}=-1.901 \mathrm{eV} \\
& \mathrm{MM}=12 \mu_{\mathrm{B}}
\end{aligned}
$$

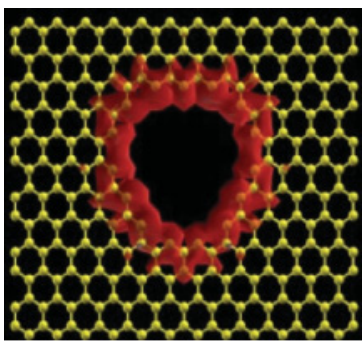

$$
\begin{aligned}
& E=-2.393 \mathrm{eV} \\
& M M=1 \mu_{B}
\end{aligned}
$$

FIG. 5. (Color online) Possible intrinsic origin of the high transition temperature in nanohole-induced magnetism exemplified by configurations 22A (upper panel) and 37A (lower panel). The energy differences relative to the nonmagnetic state and the net magnetic moments are shown. Red (blue) isosurfaces denote positive (negative) spin polarization. The isosurface value is 0.015 electrons $/ \AA^{3}$. 
edge results in a local suppression of magnetism in configurations 26B, 38A, and 55A (see Fig. 1), as was reported for nanoribbons. ${ }^{62}$ Interestingly, such local suppression may actually increase the total magnetic moments, as found in $26 \mathrm{~B}$ and 55A.

A theoretical basis for long-range RTFM in pure carbon has yet to be unambiguously established. Percolation theory ${ }^{63}$ sets a strict condition for a magnetic ground state of diluted systems: the concentration of magnetic impurity should exceed the percolation threshold for a collective magnetism. On the other hand, the strong magnetism within large nanoholes enables us to propose an alternative mechanism: namely, the observed strong ferromagnetism originates from the local magnetism induced by the nanoholes. In such a scenario, a stringent requirement concerns the size of the magnetic center; namely, the threshold for remanent, saturation magnetization, and hysteresis is a few nanometers, ${ }^{47}$ which can be readily achieved in irradiated and chemically prepared graphene samples. ${ }^{22,34}$ Small isolated magnetic regions only contribute to paramagnetism.

Another important question then arises: What is the origin of the observed high $T_{\mathrm{C}}$ ? We address this by considering configurations 22A and 37A as examples, as illustrated in Fig. 5. Each nanohole possesses several low-lying and energetically competitive magnetic solutions, most of which have nonzero net spin moments. All of these magnetic states are much lower in energy than the corresponding nonmagnetic state (by $\sim 1.9-2.1 \mathrm{eV}$ for $22 \mathrm{~A}$ and by $\sim 2.2-2.7 \mathrm{eV}$ for $37 \mathrm{~A})$. The energetics between different magnetic solutions for various nanoholes are listed in Table I. For some large nanoholes, such as 37A, all the magnetic solutions possess nonzero net magnetic moment. This means the resulted magnetization can be partially frustrated yet extremely robust against thermal fluctuation. These high stabilization energies driven by magnetic interaction (the energy difference between magnetic and nonmagnetic states) within large nanoholes explicitly imply high transition temperature in the magnetization-temperature curve, which we attribute to the experimentally observed high $T_{\mathrm{C}}$. The local nature of RTFM is in line with the experimental suggestion that only a small fraction $(\sim 0.2 \%)$ of carbon atoms contributed to the weak magnetization. ${ }^{33}$ This alternative and simple mechanism for carbon-based RTFM does not require other chemical species, and avoids the challenging requirements for distant yet strong interactions between magnetic centers. This scenario could also apply to graphite and other diluted magnetic systems. ${ }^{64,65}$ Indeed, locally accumulated, two-dimensional networks of vacancies were attributed to the RTFM in graphite. ${ }^{25}$

It is interesting to note, as shown in Table I, that for a large number of the configurations studied, there exists a significant conflict between the presently calculated magnetic moments and those predicted on the basis of Liebs theorem, ${ }^{66}$ which is a nearest-neighbor Hubbard model, and the lattice relaxation effects are not considered therein. We note this derivation holds true even for some unrelaxed structures, such as triangular nanoholes (see Table I). Such a conclusion is supported by the results in Ref. 19. These results demonstrate that a full quantum-mechanical treatment is necessary to achieve a complete, self-consistent understanding of the magnetic structures of the nanoholes in graphene.
TABLE I. Magnetic properties and band-gap values for selected nanoholes. Magnetic moment (MM) values, predicted based on Lieb's thoerem by counting the number of missing sites belonging to $A$ and $B$ sublattices, $\left(\mid N_{A}-N_{B}\right) \mid$, and the calculated ones by DFT, are compared. Total energy differences between the nonmagnetic and antiferromagnetic states $\left(\Delta E_{1}=E_{\mathrm{NM}}-E_{\mathrm{AFM}}\right)$, and between the ferromagnetic and antiferromanetic states $\left(\Delta E_{2}=E_{\mathrm{FM}}-E_{\mathrm{AFM}}\right)$ are shown, as well as the calculated energy band gap $E_{g}$. The magic nanoholes are highlighted in bold.

\begin{tabular}{|c|c|c|c|c|c|c|}
\hline Structure & $\left(N_{A}, N_{B}\right)$ & $\begin{array}{c}\left|N_{A}-N_{B}\right| \\
\mu_{\mathrm{B}} / \text { cell }\end{array}$ & $\begin{array}{c}\text { MM } \\
\left(\mu_{\mathrm{B}} / \text { cell }\right)\end{array}$ & $\begin{array}{l}\Delta E_{1} \\
(\mathrm{eV})\end{array}$ & $\begin{array}{l}\Delta E_{2} \\
(\mathrm{eV})\end{array}$ & $\begin{array}{c}E_{g} \\
(\mathrm{eV})\end{array}$ \\
\hline
\end{tabular}

\begin{tabular}{|c|c|c|c|c|c|c|}
\hline \multicolumn{7}{|c|}{ Relaxed configurations } \\
\hline $1 \mathrm{~A}$ & $(0,1)$ & 1 & 1 & \multicolumn{2}{|l|}{0.176} & 0.07 \\
\hline $\mathbf{2 A}$ & $(1,1)$ & 0 & 0 & & & 0.09 \\
\hline $4 \mathrm{~A}$ & $(3,1)$ & 2 & 0 & & & 0.11 \\
\hline 6A & $(3,3)$ & 0 & 0 & & & 0.19 \\
\hline $6 \mathrm{~B}$ & $(3,3)$ & 0 & 0 & 0.546 & 0.135 & 0.2 \\
\hline $8 \mathrm{~A}$ & $(4,4)$ & 0 & 0 & 0.825 & 0.154 & 0.23 \\
\hline $9 \mathrm{~A}$ & $(6,3)$ & 3 & 1 & 1.158 & 0.008 & 0.02 \\
\hline $16 \mathrm{~A}$ & $(8,8)$ & 0 & 0 & 0.602 & 0.128 & 0.07 \\
\hline $16 \mathrm{~B}$ & $(10,6)$ & 4 & 2 & 1.846 & 0.013 & 0.25 \\
\hline $17 \mathrm{~A}$ & $(10,7)$ & 3 & 3 & 0.763 & 0.012 & 0.04 \\
\hline $19 \mathrm{~A}$ & $(10,9)$ & 1 & 0 & & & 0.03 \\
\hline $22 \mathrm{~A}$ & $(12,10)$ & 2 & 6 & 2.292 & 0.387 & 0.13 \\
\hline $23 \mathrm{~A}$ & $(12,11)$ & 1 & 1 & 2.269 & 0.340 & 0.08 \\
\hline $23 \mathrm{~B}$ & $(13,10)$ & 3 & 6 & 2.403 & 0.276 & 0.02 \\
\hline $24 \mathrm{~A}$ & $(12,12)$ & 0 & 0 & 2.060 & 0.474 & 0.37 \\
\hline $25 \mathrm{~A}$ & $(13,12)$ & 1 & 1 & 2.212 & 0.330 & 0.14 \\
\hline $25 \mathrm{~B}$ & $(15,10)$ & 5 & 3 & 2.709 & 0.0185 & 0.16 \\
\hline $26 \mathrm{~A}$ & $(14,12)$ & 2 & 2 & 2.014 & 0.401 & 0.13 \\
\hline $26 \mathrm{~B}$ & $(15,11)$ & 4 & 6 & 1.779 & & 0.14 \\
\hline $27 \mathrm{~A}$ & $(15,12)$ & 3 & 3 & 1.034 & & 0.05 \\
\hline 28A & $(15,13)$ & 2 & 0 & & & 0.26 \\
\hline $28 \mathrm{~B}$ & $(14,14)$ & 0 & 0 & 1.251 & 0.683 & 0.17 \\
\hline $36 \mathrm{~A}$ & $(19,17)$ & 2 & 2 & 3.028 & 0.381 & 0.09 \\
\hline $36 \mathrm{~B}$ & $(21,15)$ & 6 & 4 & 2.067 & 0.054 & 0.06 \\
\hline $37 \mathrm{~A}$ & $(18,19)$ & 1 & 1 & 2.721 & 0.472 & 0.23 \\
\hline $38 \mathrm{~A}$ & $(19,19)$ & 0 & 0 & 2.210 & 0.297 & 0.14 \\
\hline $38 \mathrm{~B}$ & $(21,17)$ & 4 & 6 & 1.023 & & 0.03 \\
\hline 39A & $(21,18)$ & 3 & 3 & 0.893 & & 0.05 \\
\hline $42 \mathrm{~A}$ & $(21,21)$ & 0 & 0 & 1.286 & 0.019 & 0.45 \\
\hline $43 \mathrm{~A}$ & $(22,21)$ & 1 & 1 & 1.118 & 0.013 & 0.11 \\
\hline $44 \mathrm{~A}$ & $(22,22)$ & 0 & 0 & 0.517 & 0.023 & 0.19 \\
\hline $48 \mathrm{~A}$ & $(24,24)$ & 0 & 0 & & & 0.58 \\
\hline $49 \mathrm{~A}$ & $(25,24)$ & 1 & 1 & 0.389 & & 0.33 \\
\hline 49B & $(28,21)$ & 7 & 5 & 1.978 & 0.048 & 0.31 \\
\hline $50 \mathrm{~A}$ & $(28,22)$ & 6 & 8 & 1.872 & 0.029 & 0.34 \\
\hline $51 \mathrm{~A}$ & $(28,23)$ & 5 & 9 & 1.801 & & 0.36 \\
\hline $52 \mathrm{~A}$ & $(28,24)$ & 4 & 6 & 1.560 & & 0.41 \\
\hline $54 \mathrm{~A}$ & $(27,27)$ & 0 & 0 & 3.561 & 0.507 & 0.51 \\
\hline $55 \mathrm{~A}$ & $(28,27)$ & 1 & 3 & 2.969 & 0.385 & 0.30 \\
\hline $59 \mathrm{~A}$ & $(29,30)$ & 1 & 3 & 0.592 & & 0.10 \\
\hline \multirow[t]{2}{*}{$60 \mathrm{~A}$} & $(30,30)$ & 0 & 0 & & & 0.37 \\
\hline & \multicolumn{5}{|c|}{ Unrelaxed triangular configurations } & \\
\hline $4 \mathrm{~A}$ & $(3,1)$ & 2 & 0 & & & \\
\hline $9 \mathrm{~A}$ & $(6,3)$ & 3 & 3 & 0.595 & -0.020 & \\
\hline $16 \mathrm{~B}$ & $(10,6)$ & 4 & 6 & 1.846 & -0.418 & \\
\hline $25 \mathrm{~B}$ & $(15,10)$ & 5 & 9 & 2.090 & -0.0053 & \\
\hline
\end{tabular}


The presence of nanoholes also disrupts the sublattice symmetry of graphene and opens a semiconducting gap at the Fermi edge, ${ }^{19,67}$ which can be controlled by varying the defect concentration. ${ }^{15}$ Our results further confirm the feasibility of band-gap engineering in graphene through designed nanoholes, as shown in Fig. 4(b). Larger holes tend to exhibit larger band gaps. However, the band-gap values are also sensitive to the shape of the nanoholes. Thus, our results constitute a useful database for the selective band-gap engineering in graphene. To this end, further progress in this endeavor will require novel manufacturing techniques ${ }^{15}$ that allow control over nanohole architecture and edge configuration with atomic precision.

Stable hole structures are required for virtually all devices that incorporate nanoholes, including nanomeshes, ${ }^{15}$ antidots, ${ }^{14,18}$ metallic wire, ${ }^{21}$ and host sites of functional groups and catalytic sites. ${ }^{13}$ Thus, our finding of the stable structures with diverse properties will enable the rational design of long-lasting devices using nanoholes as the building blocks. More importantly, the integration of magnetism and semiconductivity is a key requirement for semiconductor spintronics. ${ }^{68}$ Here, the nanohole-induced, tunable magneticsemiconducting behavior may present a crucial step toward graphene-based spintronics. ${ }^{35,69}$

\section{CONCLUSION}

To summarize, extensive first-principles calculations reveal that irradiation tend to lead to the formation of large nanoholes, as observed in experiments. Ring structures and modified triangular and hexagonal nanoholes containing extra vacancies at the zigzag edge possess high stability; the magic numbers are $2,4,6,28,39,42,52$, and 60 . A large number of the nanoholes exhibit a magnetic state with a finite-energy band gap. We attribute the observed RTFM to the strong local ferri- or ferromagnetic nanoholes. Such a mechanism does not rely on external agencies. The combination of tunable semiconducting behavior and strong ferromagnetism shows promise for practical graphene-based spintronic applications through nanohole engineering in graphene.

\section{ACKNOWLEDGMENTS}

We acknowledge the computing resources provided by the National Computational Infrastructure (Australia) and support from the Australian Research Council. Support from Dr. K. R. Ratinac at the AMMRF node at the University of Sydney (ACMM) is gratefully acknowledged.
${ }^{1}$ K. S. Novoselov et al., Science 306, 666 (2004).

${ }^{2}$ K. S. Novoselov et al., Nature (London) 438, 197 (2005).

${ }^{3}$ C. Gomez-Navarro, M. Burghard, and K. Kern, Nano Lett. 8, 2045 (2008).

${ }^{4}$ A. H. C. Neto, F. Guinea, N. M. R. Peres, K. S. Novoselov, and A. K. Geim, Rev. Mod. Phys. 81, 109 (2009).

${ }^{5}$ A. K. Geim and K. S. Novoselov, Nature Mater. 6, 183 (2007).

${ }^{6}$ A. Hashimoto, K. Suenaga, A. Gloter, K. Urita, and S. Iijima, Nature (London) 430, 870 (2004).

${ }^{7}$ R. Khare, S. L. Mielke, J. T. Paci, S. Zhang, R. Ballarini, G. C. Schatz, and T. Belytschko, Phys. Rev. B 75, 075412 (2007).

${ }^{8}$ N. M. R. Peres, F. Guinea, and A. H. Castro Neto, Phys. Rev. B 73, 125411 (2006).

${ }^{9}$ S. V. Morozov, K. S. Novoselov, M. I. Katsnelson, F. Schedin, L. A. Ponomarenko, D. Jiang, and A. K. Geim, Phys. Rev. Lett. 97, 016801 (2006).

${ }^{10}$ J. H. Bardarson, J. Tworzydlo, P. W. Brouwer, and C. W. J. Beenakker, Phys. Rev. Lett. 99, 106801 (2007).

${ }^{11}$ J. H. Chen, W. G. Cullen, C. Jang, M. S. Fuhrer, and E. D. Williams, Phys. Rev. Lett. 102, 236805 (2009).

${ }^{12}$ H. J. Queisser and E. E. Haller, Science 281, 945 (1998).

${ }^{13}$ M. K. Kostov et al., Phys. Rev. Lett. 95, 136105 (2005).

${ }^{14}$ T. G. Pedersen, C. Flindt, J. Pedersen, N. A. Mortensen, A. P. Jauho, and K. Pedersen, Phys. Rev. Lett. 100, 136804 (2008).

${ }^{15}$ J. W. Bai et al., Nature Nanotech. 5, 190 (2010).

${ }^{16}$ J. I. Paredes et al., J. Phys. Chem. C 113, 10249 (2009).

${ }^{17}$ D. J. Appelhans, Z. B. Lin, and M. T. Lusk, Phys. Rev. B 82, 073410 (2010).

${ }^{18}$ J. A. Furst, T. G. Pedersen, M. Brandbyge, and A. P. Jauho, Phys. Rev. B 80, 115117 (2009).

${ }^{19}$ M. Topsakal, E. Akturk, H. Sevincli, and S. Ciraci, Phys. Rev. B 78, 235435 (2008).
${ }^{20}$ M. T. Lusk and L. D. Carr, Phys. Rev. Lett. 100, 175503 (2008).

${ }^{21}$ J. Lahiri et al., Nature Nanotech. 5, 326 (2010).

${ }^{22}$ C. O. Girit et al., Science 323, 1705 (2009).

${ }^{23}$ P. O. Lehtinen, A. S. Foster, Y. Ma, A. V. Krasheninnikov, and R. M. Nieminen, Phys. Rev. Lett. 93, 187202 (2004).

${ }^{24}$ X. M. Yang et al., Carbon 47, 1399 (2009).

${ }^{25}$ J. Cervenka, M. I. Katsnelson, and C. F. J. Flipse, Nature Phys. 5, 840 (2009).

${ }^{26}$ H. Ohldag, T. Tyliszczak, R. Hohne, D. Spemann, P. Esquinazi, M. Ungureanu, and T. Butz,Phys. Rev. Lett. 98, 187204 (2007).

${ }^{27}$ J. M. D. Coey, M. Venkatesan, C. B. Fitzgerald, A. P. Douvalis, and I. S. Sanders, Nature (London) 420, 156 (2002).

${ }^{28}$ K. H. Han et al., Adv. Mater. 15, 1719 (2003).

${ }^{29}$ P. Esquinazi, D. Spemann, R. Hohne, A. Setzer, K. H. Han, and T. Butz, Phys. Rev. Lett. 91, 227201 (2003).

${ }^{30}$ G.-D. Lee, C. Z. Wang, E. Yoon, N.-M. Hwang, D.-Y. Kim, and K. M. Ho, Phys. Rev. Lett. 95, 205501 (2005).

${ }^{31}$ K. Urita, K. Suenaga, T. Sugai, H. Shinohara, and S. Iijima, Phys. Rev. Lett. 94, 155502 (2005).

${ }^{32}$ H. Xia et al., Adv. Mater. 20, 4679 (2008).

${ }^{33}$ Y. Wang et al., Nano Lett. 9, 220 (2009).

${ }^{34}$ C. Gomez-Navarro et al., Nano Lett. 10, 1144 (2010).

${ }^{35}$ O. V. Yazyev, Rep. Prog. Phys. 73, 056501 (2010).

${ }^{36}$ O. V. Yazyev and L. Helm, Phys. Rev. B 75, 125408 (2007).

${ }^{37}$ M. M. Ugeda, I. Brihuega, F. Guinea, and J. M. Gomez-Rodriguez, Phys. Rev. Lett. 104, 096804 (2010).

${ }^{38}$ R. Singh and P. Kroll, J. Phys. Condens. Matter 21, 196002 (2009).

${ }^{39}$ D. Yu, E. M. Lupton, M. Liu, W. Liu, and F. Liu, Nano Res. 1, 56 (2008).

${ }^{40}$ D. Yu, E. M. Lupton, H. J. Gao, C. Zhang, and F. Liu, Nano Res. 1, 497 (2008).

${ }^{41}$ L. Chen, D. Yu, and F. Liu, Appl. Phys. Lett. 93, 223106 (2008). 
${ }^{42}$ E. J. Duplock, M. Scheffler, and P. J. D. Lindan, Phys. Rev. Lett. 92, 225502 (2004).

${ }^{43}$ D. W. Boukhvalov and M. I. Katsnelson, Nano Lett. 8, 4373 (2008).

${ }^{44}$ J. Zhou, Q. Wang, Q. Sun, X. S. Chen, Y. Kawazoe, and P. Jena, Nano Lett. 9, 3867 (2009).

${ }^{45}$ L. Pisani, B. Montanari, and N. M. Harrison, New J. Phys. 10, 033002 (2008).

${ }^{46}$ W. F. Li et al., Phys. Chem. Chem. Phys. 12, 13699 (2010).

${ }^{47}$ J. S. Miller and M. Drillon, Magnetism : Molecules to Materials (Wiley-VCH, Weinheim, 2001).

${ }^{48}$ J. Osorio-Guillén, S. Lany, S. V. Barabash, and A. Zunger, Phys. Rev. B 75, 184421 (2007).

${ }^{49}$ M. A. H. Vozmediano, M. P. Lopez-Sancho, T. Stauber, and F. Guinea, Phys. Rev. B 72, 155121 (2005).

${ }^{50}$ J. Y. Huang, F. Ding, B. I. Yakobson, P. Lu, L. Qi, and J. Li, Proc. Natl. Acad. Sci. USA 106, 10103 (2009).

${ }^{51}$ O. V. Yazyev, W. L. Wang, S. Meng, and E. Kaxiras, Nano Lett. 8, 766 (2008).

${ }^{52}$ L. R. Radovic and B. Bockrath, J. Am. Chem. Soc. 127, 5917 (2005).

${ }^{53}$ J. Fernandez-Rossier and J. J. Palacios, Phys. Rev. Lett. 99, 177204 (2007).

${ }^{54}$ J. Kunstmann, C. Ozdogan, A. Quandt, and H. Fehske, Phys. Rev. B 83, 045414 (2011).

${ }^{55}$ J. P. Perdew, K. Burke, and M. Ernzerhof, Phys. Rev. Lett. 77, 3865 (1996).
${ }^{56}$ B. Delley, J. Chem. Phys. 113, 7756 (2000); 92, 508 (1990).

${ }^{57}$ M. Saito, K. Yamashita, and T. Oda, Jpn. J. Appl. Phys. 46, L1185 (2007).

${ }^{58}$ See Supplemental Material at http://link.aps.org/supplemental/ 10.1103/PhysRevB.84.125410 for Supplemental Fig. 1S, showing the unrelaxed and relaxed structures considered in this study.

${ }^{59}$ J. M. Carlsson and M. Scheffler, Phys. Rev. Lett. 96, 046806 (2006).

${ }^{60}$ See Supplemental Material at http://link.aps.org/supplemental/ 10.1103/PhysRevB.84.125410 for Supplemental Fig. 2S, showing the bottom-up approach used to construct the growth pathways of the nanoholes.

${ }^{61}$ H. Zhang et al., Diam. Relat. Mater. 19, 1240 (2010).

${ }^{62}$ B. Huang, F. Liu, J. Wu, B. L. Gu, and W. Duan, Phys. Rev. B 77, 153411 (2008).

${ }^{63}$ D. Stauffer and A. Aharony, Introduction to Percolation Theory (Taylor \& Francis, London, 1994).

${ }^{64}$ X. Y. Cui, J. E. Medvedeva, A. J. Freeman, B. Delley, N. Newman, and C. Stampfl, Phys. Rev. Lett. 95, 256404 (2005); Phys. Rev. B 75, 155205 (2007)

${ }^{65}$ D. Bougeard, S. Ahlers, A. Trampert, N. Sircar, and G. Abstreiter, Phys. Rev. Lett. 97, 237202 (2006).

${ }^{66}$ E. H. Lieb, Phys. Rev. Lett. 62, 1201 (1989).

${ }^{67}$ W. Liu, Z. F. Wang, Q. W. Shi, J. L. Yang, and F. Liu, Phys. Rev. B 80, 233405 (2009).

${ }^{68}$ D. D. Awschalom and M. E. Flatte, Nature Phys. 3, 153 (2007).

${ }^{69}$ Y. W. Son, M. L. Cohen, and S. G. Louie, Nature (London) 444, 347 (2006). 